\title{
Custo-volume-lucro como ferramenta de gestão em uma empresa de serviços
}

Flávia Zoboli Dalmacio
Doutorado em Contabilidade e Controladoria pela Universidade de São Paulo -
FEA/USP
Professora da Universidade de São Paulo - FEA/USP Avenida Prof. Luciano Gualberto 908. FEA3. Cidade Universitária. São Paulo/ SP. CEP: 05508-010

E-mail: flaviazd@usp.br

Luciene Laurett Rangel Fundação Instituto Capixaba em Pesquisas em Contabilidade, Economia e Finanças FUCAPE E-mail: luciene.rangel@garoto.com.br

Venina de Almeida Lopes Mestrado em Contabilidade e Finanças pela FUCAPE Professora da Faculdade Vitoriana de Ciências Contábeis - FAVI Avenida Nossa Senhora da Penha 1.800. Barro Vermelho. Vitoria/ES. CEP: 29045-400 E-mail:venina.lopes@gmail.com

Aridelmo José Campanharo Teixeira Doutorado em Contabilidade e Controladoria pela Universidade de São Paulo - USP Professor da FUCAPE Business School Avenida Fernando Ferrari 1358. Bairro Boa Vista. Vitória/ ES. CEP: 29075-505 E-mail: aridelmo@fucape.br

\section{RESUMO}

Este artigo evidencia a aplicação de um modelo de análise de custo-volume-lucro em uma empresa prestadora de um mix de serviços, utilizando planilha eletrônica Microsoft Excel. A partir de um exemplo prático, é demonstrado como aplicar conceitos teóricos no acompanhamento do desempenho operacional de uma entidade seja ela, comercial, industrial ou prestadora de serviços, de pequeno ou de grande porte. Para tanto, desenvolveu-se um modelo de simulador operacional, com o intuito de permitir que 0 gestor efetue, de forma simples e prática, a elaboração de um planejamento interno para controle de seus processos. Salienta-se que, apesar da existência de softwares mais sofisticados no mercado, a relação custo versus benefício para empresas de pequeno e de médio porte, é um fator limitante a ser considerado.

Palavras-chave: Custo. Volume. Lucro. Gestão. Empresa de serviços. 
Custo-volume-lucro como ferramenta de gestão em uma empresa de serviços

Flávia Zoboli Dalmacio, Luciene Laurett Rangel, Venina de Almeida Lopes, Aridelmo José Campanharo

Teixeira.

Cost-volume-profit as a management tool in a service company

\section{ABSTRACT}

This article highlights the application of a model of cost-volume-profit, in a company providing a mix of services, using Microsoft Excel spreadsheet. From a practical example, it is shown how to apply theoretical concepts in monitoring the operational performance of an entity whether it be commercial, industrial or service company, small or large. To this end, we developed operating simulator model, in order to allow the manager make, so simple and practical, developing a internal planning to control their processes. It should be noted that despite the existence of more sophisticated software in the market, cost versus benefits relationship for small and medium businesses, is a limiting factor to be considered.

Keywords: Cost. Volume. Profit. Management. Service company.

\section{INTRODUÇÃO}

Os gestores, independentemente do ramo de atividade e do porte das empresas em que atuam, devem ter como ferramenta para a tomada de decisões a elaboração de um plano operacional que os auxilie no controle dos negócios. Entretanto, acredita-se que no Brasil, diferente da realidade americana ${ }^{1}$, muitos gestores ainda não utilizam esta ferramenta, tomando decisões baseadas apenas na intuição. Um bom exemplo disso é a falsa impressão de que um aumento no volume de vendas dos produtos ou serviços, conseqüentemente, aumenta o resultado da empresa.

Quando um planejamento é elaborado, observa-se que o resultado de uma empresa é conseqüência de uma combinação de custos, volumes de venda e/ou produção e mix de produtos e/ou serviços, sendo que essa análise pode ser uma ferramenta importante na gestão dos negócios. Segundo Horngren et al. (2000, p. 44),

\footnotetext{
${ }^{1}$ Segundo Horngren et al. (2000, p. 125), "pesquisas realizadas revelam o uso quase universal de orçamentos por empresas de pequeno ou médio portes".
} 
Custo-volume-lucro como ferramenta de gestão em uma empresa de serviços Flávia Zoboli Dalmacio, Luciene Laurett Rangel, Venina de Almeida Lopes, Aridelmo José Campanharo

Teixeira.

a análise de custo-volume-lucro (CVL) propicia uma ampla visão financeira do processo de planejamento. Ela examina o comportamento das receitas totais, dos custos totais e do lucro à medida que ocorre uma mudança no nível de atividade, no preço de venda ou nos custos fixos.

Contudo, a literatura a respeito da análise do custo-volume-lucro trata apenas de aplicações dos conceitos teóricos em empresas comerciais e industriais, não abordando as prestadoras de serviços. Além disso, muitos autores comentam sobre a dificuldade em se calcular o ponto de equilíbrio em empresas que oferecem múltiplos produtos. Para Wernke (2000, p. 83), "uma das dificuldades que se apresentam (sic) no cálculo do Ponto de Equilíbrio é a diversidade de produtos que compõem o mix ofertado pela empresa e as conseqüentes distintas margens de contribuição".

Martins (2001, p. 296) acrescenta que

aplicações dos conceitos de Ponto de Equilíbrio são de grande valia e de fácil entendimento e aplicação. Mas a grande restrição [...] é o fato de termos sempre trabalhado com exemplos onde havia um único produto. [...] o que não acontece quando a empresa trabalha com diversos produtos.

$\mathrm{Na}$ hipótese da existência de diversos produtos sendo elaborados pela empresa, o assunto se complica, já que os custos e despesas variáveis são diferenciados também para cada um, o que provoca a impossibilidade de cálculo de um Ponto de Equilíbrio global.

Diante do exposto, surge o questionamento: como utilizar, de forma simples e prática, a análise de custo-volume-lucro numa empresa prestadora de um mix de serviços?

Este estudo, portanto, tem por objetivo utilizar a análise de custo-volume-lucro em uma empresa prestadora de um mix de serviços e evidenciar a viabilidade do cálculo de um ponto de equilíbrio global, tendo em vista as facilidades hoje encontradas, após o surgimento/aprimoramento das planilhas eletrônicas.

Para tanto, desenvolveu-se um simulador em planilha eletrônica Microsoft Excel, com o intuito de permitir que o gestor elabore de forma simples e prática um 
Custo-volume-lucro como ferramenta de gestão em uma empresa de serviços

Flávia Zoboli Dalmacio, Luciene Laurett Rangel, Venina de Almeida Lopes, Aridelmo José Campanharo

planejamento interno para controle de seus processos. Os aspectos conceituais mais relevantes utilizados no modelo de análise de custo-volume-lucro e aplicados nesse estudo são discriminados na contextualização teórica a seguir.

\section{CONTEXTUALIZAÇÃO TEÓRICA}

A análise de custo-volume-lucro auxilia o processo de elaboração de um plano operacional. No entanto, para se discutir questões de planejamento e controle faz-se necessário um conhecimento a respeito do que são custos fixos, variáveis e mistos, pois, segundo Jiambalvo (2002, p. 76), "o gestor precisa saber como os custos se comportam, quando o nível de atividade do negócio [...] se altera".

Custos fixos são custos que não se alteram em resposta a mudanças nos níveis de atividade do negócio; já os custos variáveis aumentam ou diminuem em resposta a aumentos ou a reduções no nível de atividade; e os mistos ou semivariáveis são custos que contém tanto um elemento de custo variável quanto um elemento de custo fixo. Em muitos casos há dificuldade para identificar quanto do custo total é fixo e quanto é variável, porém existem técnicas para a estimativa destes montantes, dentre elas, destacam-se: a análise de contas; o gráfico de dispersão; o método de pontos máximo e mínimo; e a análise de regressão².

Depois de estimados os custos fixos e variáveis, a análise do custo-volume-lucro de acordo com Horngren et al. (2000, p. 45) "pode ser usada para examinar como várias alternativas de simulação levadas em consideração por um tomador de decisão afetam o lucro operacional. O ponto de equilíbrio é, freqüentemente, um item de interesse nesta análise". Este, de acordo com Atkinson et al. (2000, p. 192), é "o nível em que o volume de vendas cobre os custos fixos dos recursos comprometidos", ou seja, é o patamar acima do qual a empresa deve operar para não obter prejuízo.

O ponto de equilíbrio em quantidade pode ser obtido por meio da fórmula:

\footnotetext{
${ }^{2}$ A forma de utilização destas técnicas encontra-se no livro Contabilidade Gerencial de James Jiambalvo (2002, p. 78-82).
} 


$$
P E=\frac{C F}{M C U}
$$

Onde:

$P E=$ ponto de equilíbrio em unidades

$C F=$ custos fixos

$M C U=$ margem de contribuição unitária ${ }^{3}$

Graficamente, também se pode analisar o ponto de equilíbrio conforme apresentado na Figura 1:

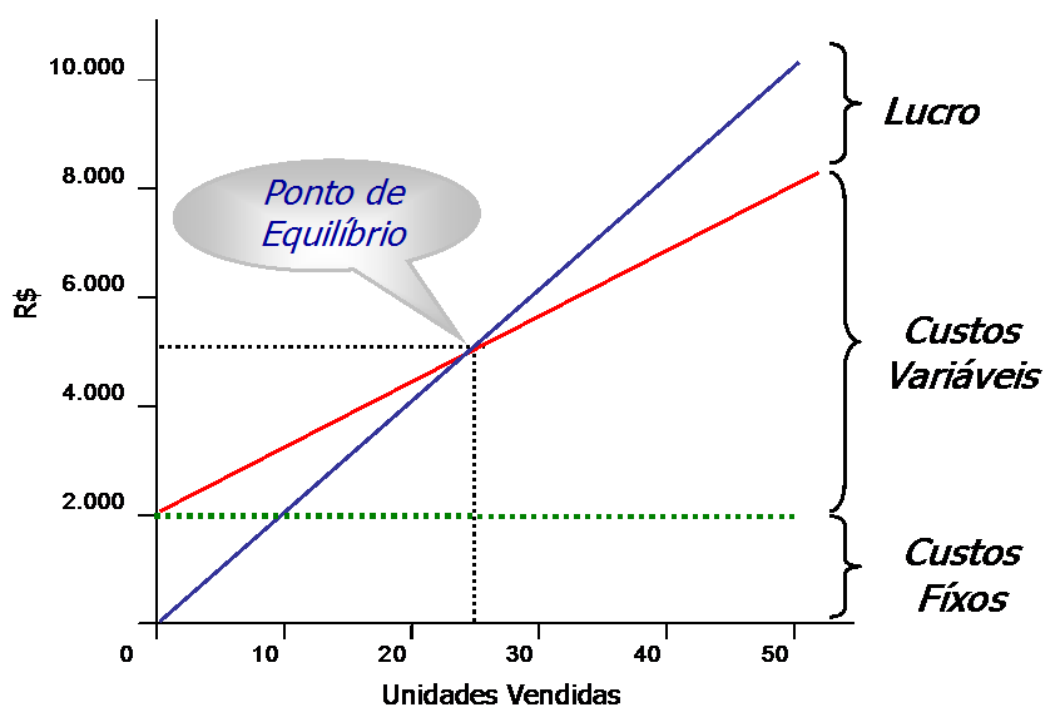

Figura 1: Gráfico do Ponto de Equilíbrio

Segundo Padoveze (apud WERNKE, 2000, p. 82), encontrando-se o ponto de equilíbrio em quantidade objetiva-se determinar a quantidade mínima que a empresa deve produzir e vender. Abaixo dessa quantidade de produção e vendas, seguramente a empresa estará operando com prejuízo.

Como não é de interesse do gestor apenas cobrir seus custos fixos e alcançar um lucro igual a zero, na elaboração do plano operacional da empresa, por meio da

\footnotetext{
${ }^{3}$ Margem de contribuição unitária é igual ao preço de venda unitário menos os custos variáveis unitários.
} 
análise de custo-volume-lucro, existe também a possibilidade de chegar-se a um resultado meta (lucro operacional a ser atingido).

Esse resultado meta pode ser obtido por meio da fórmula:

$$
R M=\frac{C F+L O}{M C U}
$$

Onde:

$R M=$ resultado meta

$C F=$ custos fixos

$\angle O=$ lucro operacional

$M C U$ = margem de contribuição unitária

Como a maioria das empresas não vende apenas um tipo de produto e/ou serviço, mas sim um portifólio, o ponto de equilíbrio pode ser obtido por meio da fórmula:

$$
P E=\frac{C F}{M C U_{m}}
$$

Onde:

$\mathrm{PE}=$ ponto de equilíbrio em unidades

$\mathrm{CF}=$ custos fixos

$\mathrm{MCU}_{\mathrm{m}}=$ margem de contribuição média ${ }^{4}$

Além disso, outra análise que pode ser elaborada por estas empresas é a classificação dos produtos e/ou serviços segundo sua rentabilidade e participação nas vendas. De acordo com Bornia (2002, p. 82), "os produtos podem ser comparados entre si de acordo com suas razões de contribuição e com sua participação nas vendas para

\footnotetext{
${ }^{4}$ Entende-se por margem de contribuição média, a margem de contribuição total dividida pela quantidade total de serviços prestados.
} 
se ter uma idéia da contribuição de cada um deles com a rentabilidade e a lucratividade da empresa".

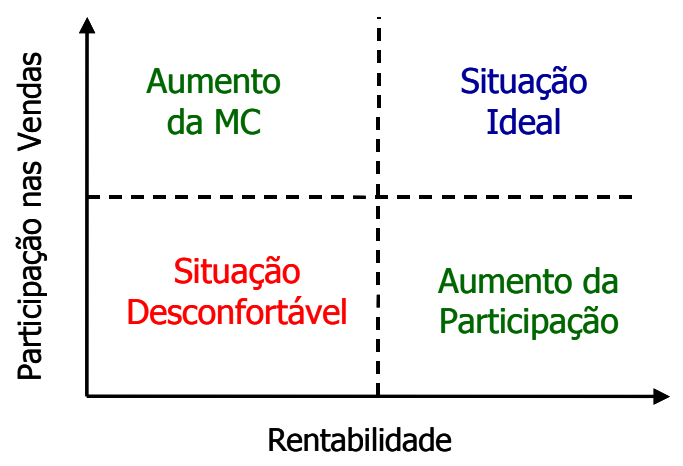

Figura 2: Classificação dos Produtos Segundo sua Rentabilidade e sua Participação nas Vendas Fonte: Adaptado de Bornia (2002, p. 82).

Conforme se observa na Figura 2, produtos e/ou serviços com alta participação nas vendas e alta rentabilidade ${ }^{5}$ são os ideais para as empresas. Produtos e/ou serviços com alta participação nas vendas e baixa rentabilidade sugerem ações para o aumento da margem de contribuição (aumento do preço unitário ou redução dos custos variáveis). Os produtos e/ou serviços com baixa participação nas vendas e alta rentabilidade sugerem ações para aumento das vendas (aumento de demanda com a manutenção dos preços). Para os produtos e/ou serviços com baixa participação nas vendas e baixa rentabilidade devem ser tomadas ações mais arrojadas, pois se apresentam em uma situação desconfortável.

Com base nestes conceitos, o gestor pode realizar um planejamento para avaliação do desempenho operacional da empresa. Aliado a isso, Maher (2001, p. 441) afirma que "fazer análise de $C V L^{6}$ em planilhas eletrônicas é particularmente útil na obtenção de respostas a questões do tipo 'e se', que aparecem no planejamento do lucro e na tomada de decisão".

\footnotetext{
${ }^{5}$ Essa rentabilidade é tratada, neste trabalho, como a margem de contribuição dos produtos e/ou serviços.

${ }^{6}$ CVL é a sigla que se refere à expressão custo-volume-lucro.
} 
Custo-volume-lucro como ferramenta de gestão em uma empresa de serviços Flávia Zoboli Dalmacio, Luciene Laurett Rangel, Venina de Almeida Lopes, Aridelmo José Campanharo

Teixeira.

O uso corrente das planilhas eletrônicas promoveu a utilização da análise de CVL em muitas organizações. Usando planilhas, os gestores podem facilmente acompanhar o CVL baseados nas análises de sensibilidade a fim de examinar o efeito e a interação das mudanças no preço de venda, nos custos variáveis unitários, nos custos fixos e no lucro operacional desejado (HORNGREN et al., 2000, p. 48, grifo nosso).

A análise de sensibilidade, segundo Horngren et al. (2000, p. 47), "é uma técnica de simulação que examina o quanto um resultado será alterado se os dados da previsão inicial não foram obtidos ou se alguma suposição fundamental for alterada".

De forma simples e prática, por meio de um exemplo hipotético, foi desenvolvido um modelo de análise de custo-volume-lucro em uma empresa prestadora de serviços, utilizando o software Microsoft Excel.

\section{MODELO DESENVOLVIDO}

Suponha que o gestor de uma empresa que presta serviços de lavagem de carros (lavajato) queira utilizar-se da análise de custo-volume-lucro na elaboração de um planejamento, para o acompanhamento do desempenho operacional do negócio. Para isso, são considerados os dados apresentados a seguir:

- Os serviços prestados pelo lavajato são: ducha, ducha executiva, lavagem de motor, lavagem geral, lavagem geral e cera, ducha e cera.

- Os custos fixos são: água (demanda), luz (demanda), salário do pessoal administrativo, arrendamento do local e depreciação dos equipamentos.

- Os custos variáveis são: água (consumo), luz (consumo), cera e comissão (salário) do pessoal operacional.

Identificadas as variáveis de decisão inseridas no processo, o gestor pode desenvolver um modelo de simulador utilizando a ferramenta excel, conforme demonstrado a seguir?

\footnotetext{
${ }^{7}$ Para a construção do simulador operacional, apresentam-se, nos apêndices 1, 2, 3 e 4, as fórmulas da planilha eletrônica.
} 
Custo-volume-lucro como ferramenta de gestão em uma empresa de serviços Flávia Zoboli Dalmacio, Luciene Laurett Rangel, Venina de Almeida Lopes, Aridelmo José Campanharo Teixeira.

\section{SIMULADOR OPERACIONAL}

A Tabela 1 apresenta uma visão global (simulador operacional, ponto de equilíbrio e demonstração de resultado) de todos os dados necessários ao funcionamento do simulador.

Tabela 1: Modelo de Simulador Operacional - Plano

\begin{tabular}{|c|c|c|c|c|c|c|c|c|c|c|}
\hline \multicolumn{11}{|c|}{ Simulador Operacional } \\
\hline Serviços & Receita & (\%) & CV & MC & (\%) & Classificação & Preço & Demanda & $\%$ & Comissão \\
\hline Ducha & 1.200 & 17 & $(219)$ & 981 & 82 & Ideal & 4,00 & 300 & 32 & 10 \\
\hline Ducha Executiva & 1.400 & 20 & $(346)$ & 1.054 & 75 & Ideal & 7,00 & 200 & 21 & 20 \\
\hline Motor & 750 & 11 & $(200)$ & 551 & 73 & $\begin{array}{c}\text { Situação } \\
\text { Desconfortável }\end{array}$ & 5,00 & 150 & 16 & 20 \\
\hline Geral & 1.500 & 21 & (333) & 1.167 & 78 & Ideal & 15,00 & 100 & 11 & 20 \\
\hline Geral e Cera & 1.190 & 17 & $(343)$ & 847 & 71 & Aumentar MC & 17,00 & 70 & 7 & 20 \\
\hline Ducha e Cera & 1.080 & 15 & $(396)$ & 684 & 63 & $\begin{array}{c}\text { Situação } \\
\text { Desconfortável }\end{array}$ & 9,00 & 120 & 13 & 20 \\
\hline Total & 7.120 & 100 & $(1.836)$ & 5.284 & 74 & Lucro Operaci & ional & & & $\mathbf{R} \$ 2.849$ \\
\hline \multicolumn{8}{|c|}{ Demonstração de Resultado } & \multicolumn{3}{|c|}{ Ponto de Equilíbrio } \\
\hline \multicolumn{4}{|c|}{ Quantidade de Carros Mês } & & & 940 & $\%$ & 433 & & $\%$ \\
\hline \multicolumn{4}{|c|}{ Quantidade de Carros dia } & & & 36 & \multicolumn{4}{|c|}{17} \\
\hline \multicolumn{2}{|l|}{ Receita Total } & & & & & $7.120,0$ & 100,0 & $3.281,0$ & & 100,0 \\
\hline \multicolumn{2}{|l|}{ Custos Variáveis } & & & & & $(1.835,9)$ & $(25,8)$ & $(846,0)$ & & $(25,8)$ \\
\hline \multicolumn{2}{|c|}{ Despesas Variáveis } & & & & & $(531,9)$ & $(7,5)$ & $(245,1)$ & & $(7,5)$ \\
\hline \multicolumn{2}{|l|}{ Água } & & & \multicolumn{2}{|c|}{$\mathrm{R} \$(0,20$} & $(188,0)$ & $(2,6)$ & $(86,6)$ & & $(2,6)$ \\
\hline \multicolumn{2}{|l|}{ Luz } & & & \multicolumn{2}{|c|}{$\mathrm{R} \$(0,13)$} & $(122,2)$ & $(1,7)$ & $(56,3)$ & & $(1,7)$ \\
\hline \multicolumn{2}{|l|}{ Cera } & & & \multicolumn{2}{|c|}{$\mathrm{R} \$(1,17)$} & $(221,7)$ & $(3,1)$ & $(102,1)$ & & $(3,1)$ \\
\hline \multicolumn{2}{|l|}{ Comissão } & & & \multirow{2}{*}{\multicolumn{2}{|c|}{$\mathrm{R} \$(0,40)$}} & $(1.304,0)$ & $(18,3)$ & $(600,9)$ & & $(18,3)$ \\
\hline Ducha & & & & & & $(120,0)$ & $(1,7)$ & $(55,3)$ & & $(1,7)$ \\
\hline \multicolumn{2}{|l|}{ Ducha Executiva } & & & \multicolumn{2}{|c|}{$\mathrm{R} \$(1,40)$} & $(280,0)$ & $(3,9)$ & $(129,0)$ & & $(3,9)$ \\
\hline
\end{tabular}


Custo-volume-lucro como ferramenta de gestão em uma empresa de serviços Flávia Zoboli Dalmacio, Luciene Laurett Rangel, Venina de Almeida Lopes, Aridelmo José Campanharo Teixeira.

\begin{tabular}{|lccccc|} 
Motor & $\mathrm{R} \$(1,00)$ & $(150,0)$ & $(2,1)$ & $(69,1)$ & $(2,1)$ \\
Geral & $\mathrm{R} \$(3,00)$ & $(300,0)$ & $(4,2)$ & $(138,2)$ & $(4,2)$ \\
Geral e Cera & $\mathrm{R} \$(3,40)$ & $(238,0)$ & $(3,3)$ & $(109,7)$ & $(3,3)$ \\
Ducha e Cera & $\mathrm{R} \$(1,80)$ & $(216,0)$ & $(3,0)$ & $(99,5)$ & $(3,0)$ \\
& & & & & \\
\hline Margem Contribuição & $\mathbf{R} \mathbf{5 , 6 2}$ & $\mathbf{5 . 2 8 4 , \mathbf { 1 }}$ & $\mathbf{7 4 , 2}$ & $\mathbf{2 . 4 3 5 , 0}$ & $\mathbf{7 4 , 2}$ \\
\hline Custo Fixo & & & & \\
Água & $\mathbf{( 2 . 4 3 5 , 0 )}$ & $\mathbf{( 3 4 , 2 )}$ & $\mathbf{( 2 . 4 3 5 , 0 )}$ & $\mathbf{( 7 4 , 2}$ \\
Luz & $(15,0)$ & $(0,2)$ & $(15,0)$ & $(0,5)$ \\
Salário Administrativo & $(20,0)$ & $(0,3)$ & $(20,0)$ & $(0,6)$ \\
Arrendamento do Local & $(900,0)$ & $(12,6)$ & $(900,0)$ & $(27,4)$ \\
Depreciação - Equipamentos & & $(1.000,0)$ & $(14,0)$ & $(1.000,0)$ & $(30,5)$ \\
& & $(500,0)$ & $(7,0)$ & $(500,0)$ & $(15,2)$ \\
\hline Resultado antes do IR & & & & $\mathbf{0}, 0$ \\
\hline
\end{tabular}

No simulador operacional, a simulação pode ser efetuada a partir da alteração dos valores de preço, demanda e comissão que se encontra em destaque (fundo em negrito). O plano a ser traçado pelo gestor no simulador dependerá do ambiente no qual a empresa está inserida.

A coluna identificada como classificação, vai se alterando, automaticamente, em conseqüência das mudanças de cenários. Isso facilita a visão do gestor para a tomada de alguma decisão com relação ao mix de serviços da empresa, pois apresenta a classificação dos serviços segundo sua margem de contribuição e sua participação nas vendas. Essa classificação é a sugerida por Bornia (2002), conforme Figura 2.

O ponto de equilíbrio pode ser modificado à medida que há alterações no mix de serviços e suas variáveis. Portanto, não há uma única quantidade de unidades para atingir-se o ponto de equilíbrio em situações de múltiplos serviços.

No caso do exemplo citado, com base na informação do ponto de equilíbrio, o gestor tem conhecimento de quantos carros precisam ser lavados no mês para cobrir seus custos totais. A Figura 3 mostra que no $12^{\circ}$ (décimo segundo) dia a empresa atinge seu ponto de equilíbrio, e a partir daí já obtém um resultado operacional positivo. 
Custo-volume-lucro como ferramenta de gestão em uma empresa de serviços Flávia Zoboli Dalmacio, Luciene Laurett Rangel, Venina de Almeida Lopes, Aridelmo José Campanharo

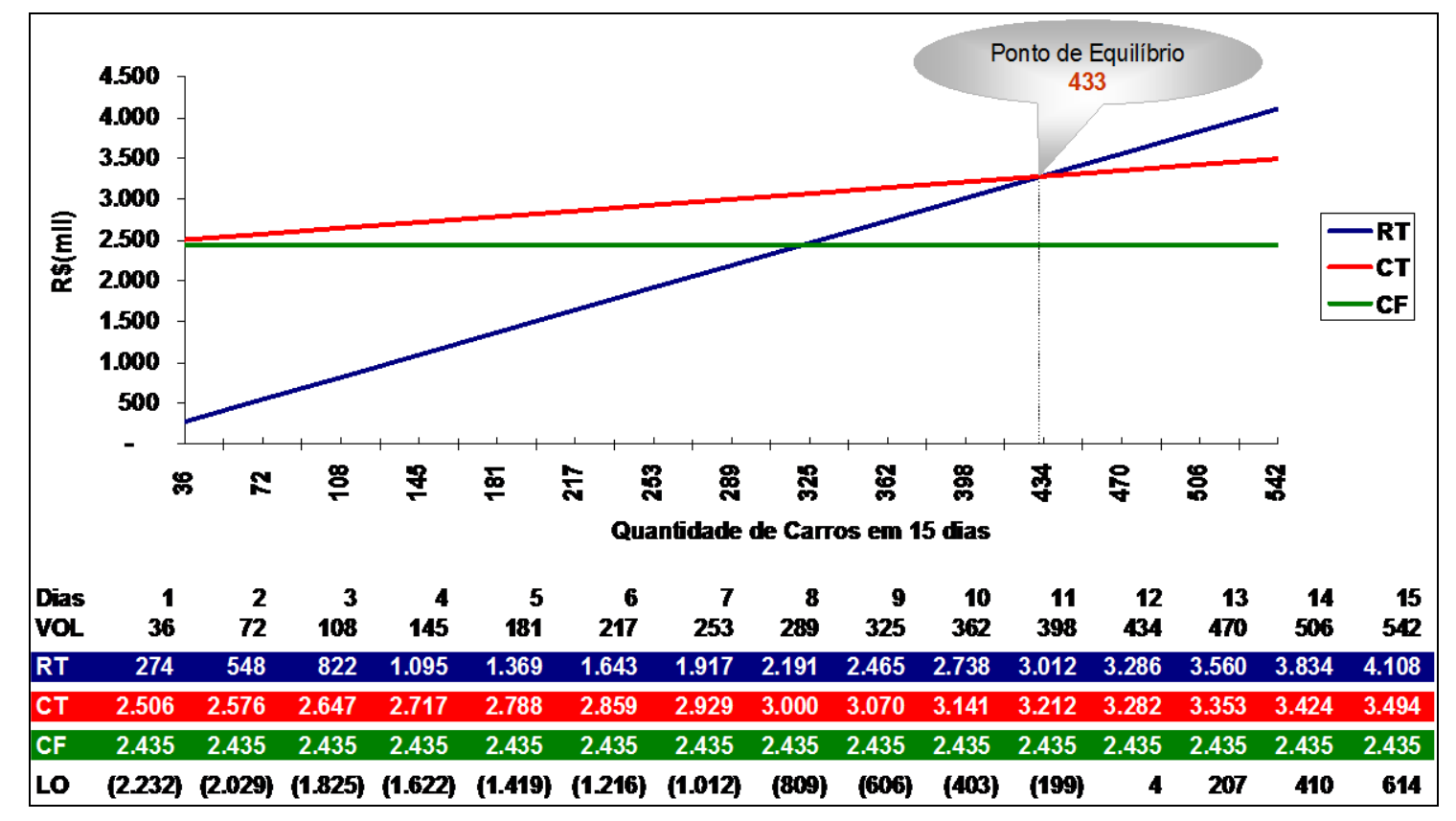

Figura 3: Gráfico do Ponto de Equilíbrio

$\mathrm{Na}$ demonstração do resultado $^{8}$, além da receita total, apresenta-se o detalhamento dos custos e despesas, fixos e variáveis, identificando-se o resultadometa (lucro operacional a ser alcançado) da empresa por meio de simulações.

Como o exemplo trata de uma empresa prestadora de um mix de serviços, mesmo que este seja modificado, as metas de receitas ainda podem ser atingidas. Entretanto, os efeitos sobre o lucro dependem de como as proporções de margem de contribuição dos produtos se alteram.

Feitas todas as possíveis simulações por meio do simulador operacional, escolhe-se um cenário e define-se um resultado meta a ser alcançado. Neste exemplo, o resultado meta considerado é o demonstrado na Tabela 1 (lucro operacional de $\mathrm{R} \$ 2.849,10)$, que serve de base para a comparação com um resultado efetivo e para o acompanhamento do desempenho operacional de vendas e custos.

\footnotetext{
${ }^{8}$ Não foram considerados, neste trabalho, os impostos e contribuições incidentes sobre os serviços prestados, com o intuito de simplificação.
} 
Custo-volume-lucro como ferramenta de gestão em uma empresa de serviços Flávia Zoboli Dalmacio, Luciene Laurett Rangel, Venina de Almeida Lopes, Aridelmo José Campanharo

A partir do momento em que a empresa tem o seu resultado realizado, o gestor pode identificar as variações ocorridas entre o resultado efetivo e o planejado, conforme demonstrado na Tabela 2.

Tabela 2: Modelo de Simulador Operacional - Efetivo

\begin{tabular}{|c|c|c|c|c|c|c|c|c|c|c|}
\hline \multicolumn{11}{|c|}{ Simulador Operacional } \\
\hline Serviços & Receita & $(\%)$ & CV & MC & $(\%)$ & Classificação & Preço & Demanda & $\%$ & Comissão \\
\hline Ducha & 921 & 12 & (263) & 658 & 71 & $\begin{array}{l}\text { Situação } \\
\text { Desconfortável }\end{array}$ & 3,50 & 263 & 29 & 16 \\
\hline $\begin{array}{l}\text { Ducha } \\
\text { Executiva }\end{array}$ & 900 & 12 & $(210)$ & 690 & 77 & $\begin{array}{l}\text { Aumentar } \\
\text { Participação }\end{array}$ & 6,00 & 150 & 17 & 16 \\
\hline Motor & 500 & 7 & $(124)$ & 376 & 75 & \begin{tabular}{|l} 
Aumentar \\
Participação
\end{tabular} & 5,00 & 100 & 11 & 16 \\
\hline Geral & 2.100 & 27 & $(402)$ & 1.698 & 81 & Ideal & 14,00 & 150 & 17 & 16 \\
\hline $\begin{array}{ll}\text { Geral } & \text { e } \\
\text { Cera } & \\
\end{array}$ & 1.952 & 25 & $(539)$ & 1.413 & 72 & Aumentar MC & 16,00 & 122 & 14 & 16 \\
\hline $\begin{array}{l}\text { Ducha e } \\
\text { Cera }\end{array}$ & 1.296 & 17 & $(408)$ & 888 & 69 & Aumentar MC & 12,00 & 108 & 12 & 16 \\
\hline Total & 7.669 & 100 & $(1.946)$ & 5.723 & 75 & Lucro Operac & ional & & & $\mathbf{R} \$ 3.288$ \\
\hline \multicolumn{8}{|c|}{ Demonstração de Resultado } & \multicolumn{3}{|c|}{ Ponto de Equilíbrio } \\
\hline \multicolumn{5}{|c|}{ Quantidade de Carros Mês } & & 893 & & 380 & & $\%$ \\
\hline \multicolumn{5}{|c|}{ Quantidade de Carros dia } & & 34 & & 15 & & \\
\hline \multicolumn{5}{|c|}{ Receita Total } & & $.668,5$ & & $3.262,9$ & & 100,0 \\
\hline \multicolumn{5}{|c|}{ Custos Variáveis } & & $.945,7)$ & & $(827,9)$ & & $(25,4)$ \\
\hline \multicolumn{5}{|c|}{ Despesas Variáveis } & & $718,8)$ & & $(305,8)$ & & $(9,4)$ \\
\hline \multicolumn{3}{|c|}{ Água } & $\mathrm{R} \$$ & $(0,28)$ & & $250,0)$ & & $(106,4)$ & & $(3,3)$ \\
\hline \multicolumn{3}{|l|}{ Luz } & $\mathrm{R} \$$ & $(0,16)$ & & $142,9)$ & & $(60,8)$ & & $(1,9)$ \\
\hline \multicolumn{3}{|l|}{ Cera } & $\mathrm{R} \$$ & $(1,42)$ & & $325,8)$ & & $(138,6)$ & & $(4,2)$ \\
\hline \multicolumn{3}{|l|}{ Comissão } & & & & $.227,0)$ & & $(522,1)$ & & $(16,0)$ \\
\hline \multicolumn{3}{|l|}{ Ducha } & $\mathrm{R} \$$ & $(0,56)$ & & $147,3)$ & & $(62,7)$ & & $(1,9)$ \\
\hline \multicolumn{3}{|c|}{ Ducha Executiva } & $\mathrm{R} \$$ & $(0,96)$ & & $144,0)$ & & $(61,3)$ & & $(1,9)$ \\
\hline \multicolumn{3}{|l|}{ Motor } & $\mathrm{R} \$$ & $(0,80)$ & & $(80,0)$ & & $(34,0)$ & & $(1,0)$ \\
\hline \multicolumn{3}{|l|}{ Geral } & $\mathrm{R} \$$ & $(2,24)$ & & $336,0)$ & & $(143,0)$ & & $(4,4)$ \\
\hline \multicolumn{3}{|c|}{ Geral e Cera } & $\mathrm{R} \$$ & $(2,56)$ & & $312,3)$ & & $(132,9)$ & & $(4,1)$ \\
\hline \multicolumn{3}{|c|}{ Ducha e Cera } & $\mathrm{R} \$$ & $(1,92)$ & & $207,4)$ & & $(88,2)$ & & $(2,7)$ \\
\hline
\end{tabular}




$\begin{array}{lccccc}\text { Margem Contribuição } & \mathbf{R} \$ \mathbf{6 , 4 1} & \mathbf{5 . 7 2 2 , 8} & \mathbf{7 4 , 6} & \mathbf{2 . 4 3 5 , 0} & \mathbf{7 4 , 6} \\ \text { Custo Fixo } & & & & \\ \text { Água } & \mathbf{( 2 . 4 3 5 , 0 )} & \mathbf{( 3 1 , 8 )} & \mathbf{( 2 . 4 3 5 , 0 )} & \mathbf{( 7 4 , 6 )} \\ \text { Luz } & (15,0) & (0,2) & (15,0) & (0,5) \\ \text { Salário Administrativo } & (20,0) & (0,3) & (20,0) & (0,6) \\ \text { Arrendamento do Local } & (900,0) & (11,7) & (900,0) & (27,6) \\ \text { Depreciação - Equipamentos } & (1.000,0) & (13,0) & (1.000,0) & (30,6) \\ & (500,0) & (6,5) & (500,0) & (15,3) \\ \text { Resultado antes do IR } & & & & \mathbf{0 , 0} & \mathbf{0 , 0}\end{array}$

Analisando a coluna de classificação do simulador operacional, observa-se que, apesar da situação desconfortável do serviço ducha, este apresenta margem de contribuição positiva, absorvendo uma parcela dos custos fixos, contribuindo com o resultado empresarial.

Outra análise que pode ser efetuada é o comparativo da demonstração de resultado realizado $x$ planejado, conforme Tabela 3 , onde se identificam as variações das receitas e dos custos fixos e variáveis. Todavia, nessa observação não fica evidente qual o serviço que contribuiu para o ganho ou para a perda do resultado operacional. 
Custo-volume-lucro como ferramenta de gestão em uma empresa de serviços Flávia Zoboli Dalmacio, Luciene Laurett Rangel, Venina de Almeida Lopes, Aridelmo José Campanharo

Tabela 3: Demonstrativo de Resultado do Lavajato - Efetivo x Plano

\begin{tabular}{|c|c|c|c|c|c|c|c|c|c|}
\hline \multicolumn{4}{|c|}{ Efetivo } & \multicolumn{3}{|c|}{ Plano Operacional } & \multicolumn{3}{|c|}{ Variação } \\
\hline \multicolumn{2}{|c|}{ Quantidade de Carros Mês } & 893 & $\%$ & & 940 & $\%$ & & (47) & $\%$ \\
\hline \multicolumn{2}{|c|}{ Quantidade de Carros dia } & 34 & & & 36 & & & (2) & \\
\hline \multicolumn{2}{|l|}{ Receita Total } & $7.668,5$ & 100,0 & & $7.120,0$ & 100,0 & & 548,5 & $\mathbf{7 , 7}$ \\
\hline \multicolumn{2}{|l|}{ Custos Variáveis } & $(1.945,7)$ & $(25,4)$ & & $(1.835,9)$ & $(23,9)$ & & $(109,8)$ & $(6,0)$ \\
\hline \multicolumn{2}{|l|}{ Desp. Variáveis } & $(718,8)$ & $(9,4)$ & & $(531,9)$ & $(6,9)$ & & $(186,9)$ & $(35,1)$ \\
\hline Água & $\mathrm{R} \$(0,28)$ & $(250,0)$ & $(3,3)$ & $\mathrm{R} \$(0,20)$ & $(188,0)$ & $(2,5)$ & $\mathrm{R} \$(0,08)$ & $(62,0)$ & $(33,0)$ \\
\hline Luz & $\mathrm{R} \$(0,16)$ & $(142,9)$ & $(1,9)$ & $R \$(0,13)$ & $(122,2)$ & $(1,6)$ & $\mathrm{R} \$(0,03)$ & $(20,7)$ & $(16,9)$ \\
\hline Cera & $\mathrm{R} \$(1,42)$ & $(325,8)$ & $(4,2)$ & $\mathrm{R} \$(1,17)$ & $(221,7)$ & $(2,9)$ & $\mathrm{R} \$(0,25)$ & $(104,2)$ & $(47,0)$ \\
\hline \multicolumn{2}{|l|}{ Comissão } & $(1.227,0)$ & $(16,0)$ & & $(1.304,0)$ & $(17,0)$ & & 77,0 & 5,9 \\
\hline Ducha & $\mathrm{R} \$(0,56)$ & $(147,3)$ & $(1,9)$ & $\mathrm{R} \$(0,40)$ & $(120,0)$ & $(1,6)$ & $\mathrm{R} \$(0,16)$ & $(27,3)$ & $(22,7)$ \\
\hline Ducha Executiva & $\mathrm{R} \$(0,96)$ & $(144,0)$ & $(1,9)$ & $\mathrm{R} \$(1,40)$ & $(280,0)$ & $(3,7)$ & $\mathrm{R} \$ 0,44$ & 136,0 & 48,6 \\
\hline Motor & $\mathrm{R} \$(0,80)$ & $(80,0)$ & $(1,0)$ & $\mathrm{R} \$(1,00)$ & $(150,0)$ & $(2,0)$ & $R \$ 0,20$ & 70,0 & 46,7 \\
\hline Geral & $\mathrm{R} \$(2,24)$ & $(336,0)$ & $(4,4)$ & $\mathrm{R} \$(3,00)$ & $(300,0)$ & $(3,9)$ & $\mathrm{R} \$ 0,76$ & $(36,0)$ & $(12,0)$ \\
\hline Geral e Cera & $\mathrm{R} \$(2,56)$ & $(312,3)$ & $(4,1)$ & $R \$(3,40)$ & $(238,0)$ & $(3,1)$ & $\mathrm{R} \$ 0,84$ & $(74,3)$ & $(31,2)$ \\
\hline Ducha e Cera & $\mathrm{R} \$(1,92)$ & $(207,4)$ & $(2,7)$ & $\mathrm{R} \$(1,80)$ & $(216,0)$ & $(2,8)$ & $\mathrm{R} \$(0,12)$ & 8,6 & 4,0 \\
\hline Margem Contrib. & $R \$ 6,41$ & $5.722,8$ & 74,6 & $\mathbf{R} \$ 5,62$ & $5.284,1$ & 68,9 & $\mathbf{R} \$ 0,79$ & 438,7 & 8,3 \\
\hline Custo Fixo & & $(2.435,0)$ & $(31,8)$ & & $(2.435,0)$ & $(31,8)$ & & 0,0 & 0,0 \\
\hline Água & & $(15,0)$ & $(0,2)$ & & $(15,0)$ & $(0,2)$ & & 0,0 & 0,0 \\
\hline Luz & & $(20,0)$ & $(0,3)$ & & $(20,0)$ & $(0,3)$ & & 0,0 & 0,0 \\
\hline Salário Administrati & & $(900,0)$ & $(11,7)$ & & $(900,0)$ & $(11,7)$ & & 0,0 & 0,0 \\
\hline Arrendamento do Lc & & $(1.000,0)$ & $(13,0)$ & & $(1.000,0)$ & $(13,0)$ & & 0,0 & 0,0 \\
\hline Depreciação - Equi & & $(500,0)$ & $(6,5)$ & & $(500,0)$ & $(6,5)$ & & 0,0 & 0,0 \\
\hline Result. antes IR & & $3.287,8$ & 42,9 & & $2.849,1$ & 37,2 & & 438,7 & 15,4 \\
\hline
\end{tabular}

Verifica-se que a empresa não conseguiu atingir a demanda esperada de 940 carros/mês, entretanto seu resultado operacional antes do imposto de renda superou 0 plano em $\mathrm{R} \$ 438,70$. Isto se deve a diversas alterações no volume, no preço e no mix de seus serviços, que são apresentadas e comentadas a seguir. 
Custo-volume-lucro como ferramenta de gestão em uma empresa de serviços Flávia Zoboli Dalmacio, Luciene Laurett Rangel, Venina de Almeida Lopes, Aridelmo José Campanharo

Tabela 4: Performance Operacional de Vendas $x$ Custos ${ }^{i}$

\begin{tabular}{l|c|c|c|c|c|c|c|c|c}
\hline \multirow{2}{*}{ Serviços } & \multicolumn{3}{|c|}{ Análise das Vendas } & \multicolumn{3}{c|}{ Análise dos Custos } & \multicolumn{3}{c}{ Análise da Margem Bruta } \\
\cline { 2 - 10 } & $\begin{array}{l}\text { Volume } \\
\text { Mix }\end{array}$ & Preço & Total & $\begin{array}{c}\text { Volume } \\
\text { Mix }\end{array}$ & Preço & Total & $\begin{array}{c}\text { Volume } \\
\text { Mix }\end{array}$ & Preço & Total \\
\hline Ducha & $(129,5)$ & $(150,0)$ & $(279,5)$ & 37,0 & $(81,0)$ & $(44,0)$ & $(92,5)$ & $(231,0)$ & $\mathbf{( 3 2 3 , 5 )}$ \\
\hline $\begin{array}{l}\text { Ducha } \\
\text { Executiva }\end{array}$ & $(300,0)$ & $(200,0)$ & $(500,0)$ & 70,0 & 66,0 & 136,0 & $(230,0)$ & $(134,0)$ & $(\mathbf{3 6 4 , 0})$ \\
\hline Motor & $(250,0)$ & 0,0 & $(250,0)$ & 62,0 & 13,5 & 75,5 & $(188,0)$ & 13,5 & $(\mathbf{1 7 4 , 5 )}$ \\
\hline Geral & 700,0 & $(100,0)$ & 600,0 & $(134,0)$ & 65,0 & $(69,0)$ & 566,0 & $(35,0)$ & $\mathbf{5 3 1 , 0}$ \\
\hline Geral e Cera & 832,0 & $(70,0)$ & 762,0 & $(229,7)$ & 33,6 & $(196,1)$ & 602,3 & $(36,4)$ & $\mathbf{5 6 5 , 9}$ \\
\hline $\begin{array}{l}\text { Ducha } \\
\text { Cera }\end{array}$ & $(144,0)$ & 360,0 & 216,0 & 45,3 & $(57,6)$ & $(12,3)$ & $(98,7)$ & 302,4 & $\mathbf{2 0 3 , 7}$ \\
\hline TOTAL & $\mathbf{7 0 8 , 5}$ & $(\mathbf{1 6 0 , 0 )}$ & $\mathbf{5 4 8 , 5}$ & $\mathbf{( 1 4 9 , 3 )}$ & $\mathbf{3 9 , 5}$ & $\mathbf{( 1 0 9 , 8 )}$ & $\mathbf{5 5 9 , 2}$ & $\mathbf{( 1 2 0 , 5 )}$ & $\mathbf{4 3 8 , 7}$ \\
\hline
\end{tabular}

A análise do desempenho operacional de vendas explica o ganho de $R \$ 548,50$ da receita total. Os serviços: lavagem geral, lavagem geral e cera, e ducha e cera são os que contribuíram para este ganho. Sendo que os dois primeiros serviços ganharam no volume ( $R \$ 700,00$ e $R \$ 832,00$, respectivamente) e o último ganhou no preço $(\mathrm{R} \$ 360,00)$, compensando, dessa forma, as perdas ocorridas nos serviços ducha, ducha executiva e lavagem de motor.

$\mathrm{Na}$ análise do desempenho operacional de custos identifica-se uma perda no volume-mix de $R \$ 149,30$ contra um ganho no preço de $R \$ 39,50$. Apesar da demanda pelos serviços da empresa ter sido menor do que o plano houve perda no volume em decorrência do mix. Ressalta-se que o acréscimo no volume dos serviços, lavagem geral e lavagem geral e cera, acarretou um aumento, em valor, na comissão dos funcionários, pois o preço destes serviços é bem superior aos demais. Aliado a isso houve um acréscimo nos preços das despesas variáveis, água, luz e cera.

A combinação da análise do desempenho operacional de vendas com a de custos justifica o ganho de $R \$ 438,70$ na margem de contribuição total e resultado operacional, pois os custos fixos se mantiveram inalterados. 
Custo-volume-lucro como ferramenta de gestão em uma empresa de serviços

Flávia Zoboli Dalmacio, Luciene Laurett Rangel, Venina de Almeida Lopes, Aridelmo José Campanharo

Teixeira.

\section{CONSIDERAÇÕES FINAIS}

Neste estudo apresenta-se um modelo de simulador operacional que permite a aplicação da análise do custo-volume-lucro em uma empresa prestadora de um mix de serviços, propiciando um melhor controle e gestão dos negócios.

Este simulador também pode ser utilizado por empresas comerciais e industriais, de pequeno e grande porte. No entanto, as análises do gestor não devem se limitar apenas aos dados gerados por esta ferramenta, devendo levar em consideração, por exemplo, o ambiente empresarial em que a empresa está inserida.

Complementando a análise de sensibilidade oferecida pelo simulador, torna-se interessante observar a estrutura de custos fixos e variáveis, controlando o nível de alavancagem operacional ${ }^{9}$ que se deseja obter, reduzindo com isso o risco inerente do negócio.

Deve-se salientar que no mercado existem softwares mais sofisticados, entretanto, a relação custo versus benefício para empresas de pequeno e médio porte é um fator limitante. Finalmente, esse estudo apresenta uma solução viável de como contornar este problema, de forma simples e prática, com o auxílio da planilha eletrônica Microsoft Excel.

\section{REFERÊNCIAS}

ATKINSON, Antony A. et al. (2000). Contabilidade gerencial. Tradução André Olímpio Mosselman Du Chenoy Castro. São Paulo: Atlas.

BORNIA, Antonio Cezar. (2002). Análise gerencial de custos: aplicação em empresas modernas. Porto Alegre: Bookman.

HORNGREN, Charles T.; FOSTER, George; DATAR, Srikant M. (2000). Contabilidade de custos. 9. ed. Tradução José Luiz Paravato. Rio de Janeiro: LTC.

JIAMBALVO, James. (2002). Contabilidade gerencial. Rio de Janeiro: LTC.

\footnotetext{
${ }^{9}$ Segundo Jiambalvo (2002, p. 87), "a alavancagem operacional relaciona-se no (sic) nível de custos fixos versus variáveis, na estrutura de custos de uma empresa. As empresas que possuem altos níveis de custos fixos possuem uma alta alavancagem operacional".
} 
MAHER, Michael. (2001). Contabilidade de custos: criando valor para a administração. Tradução José Evaristo dos Santos. São Paulo: Atlas.

MARTINS, Eliseu. (2001). Contabilidade de custos. (8. ed.). São Paulo: Atlas.

WERNKE, Rodney. (2000). Ponto de equilíbrio: considerações e comentários. Revista Brasileira de Contabilidade, Brasília, n. 125, set./out. p. 79-86.

Data de Submissão: 06/10/2011

Data de Aceite: 03/04/2013 
Custo-volume-lucro como ferramenta de gestão em uma empresa de serviços

Flávia Zoboli Dalmacio, Luciene Laurett Rangel, Venina de Almeida Lopes, Aridelmo José Campanharo

Teixeira.

Apêndice 1: Parte 1 - Simulador Operacional.

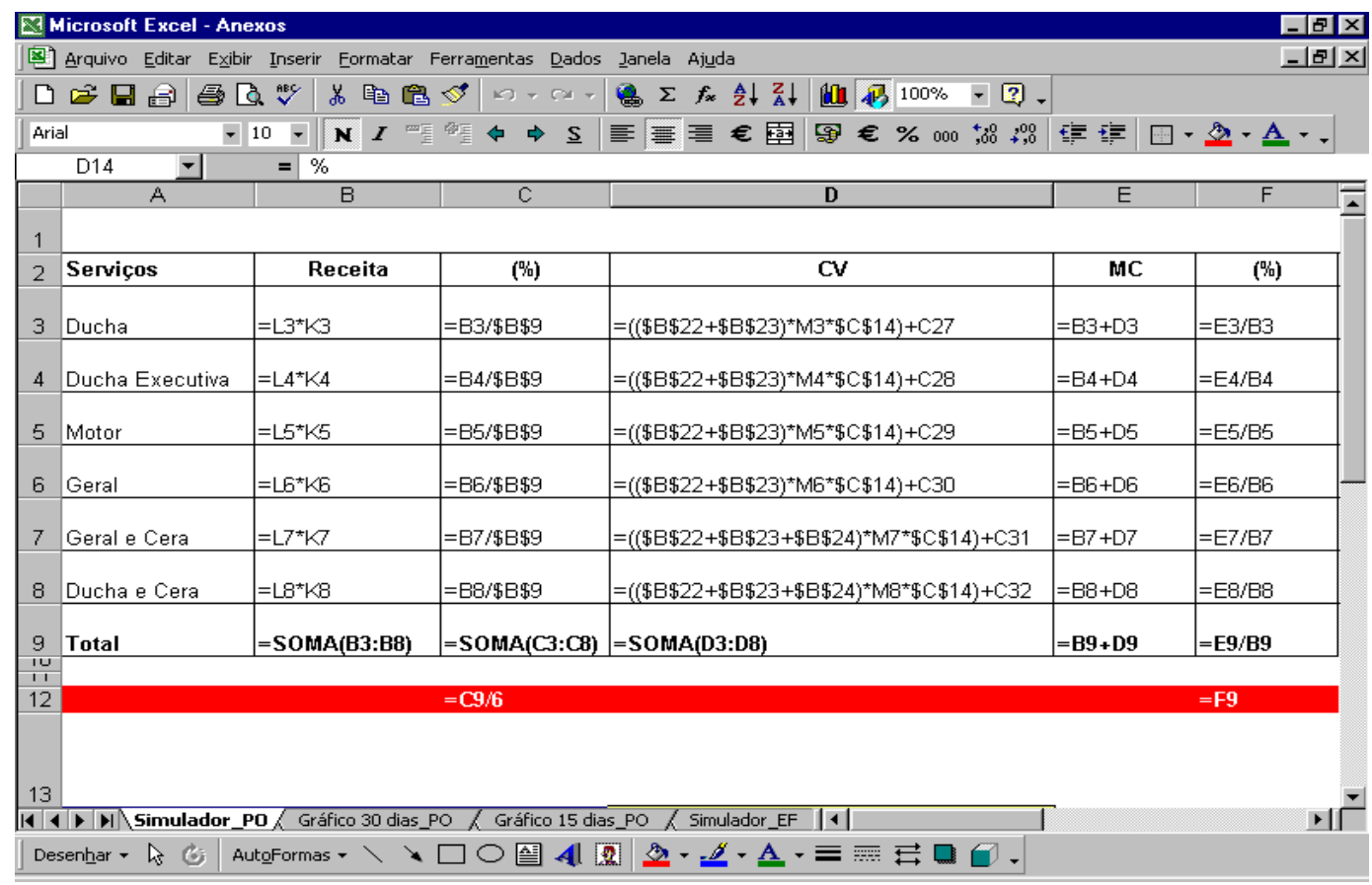

Apêndice 2: Parte 2 - Simulador Operacional.

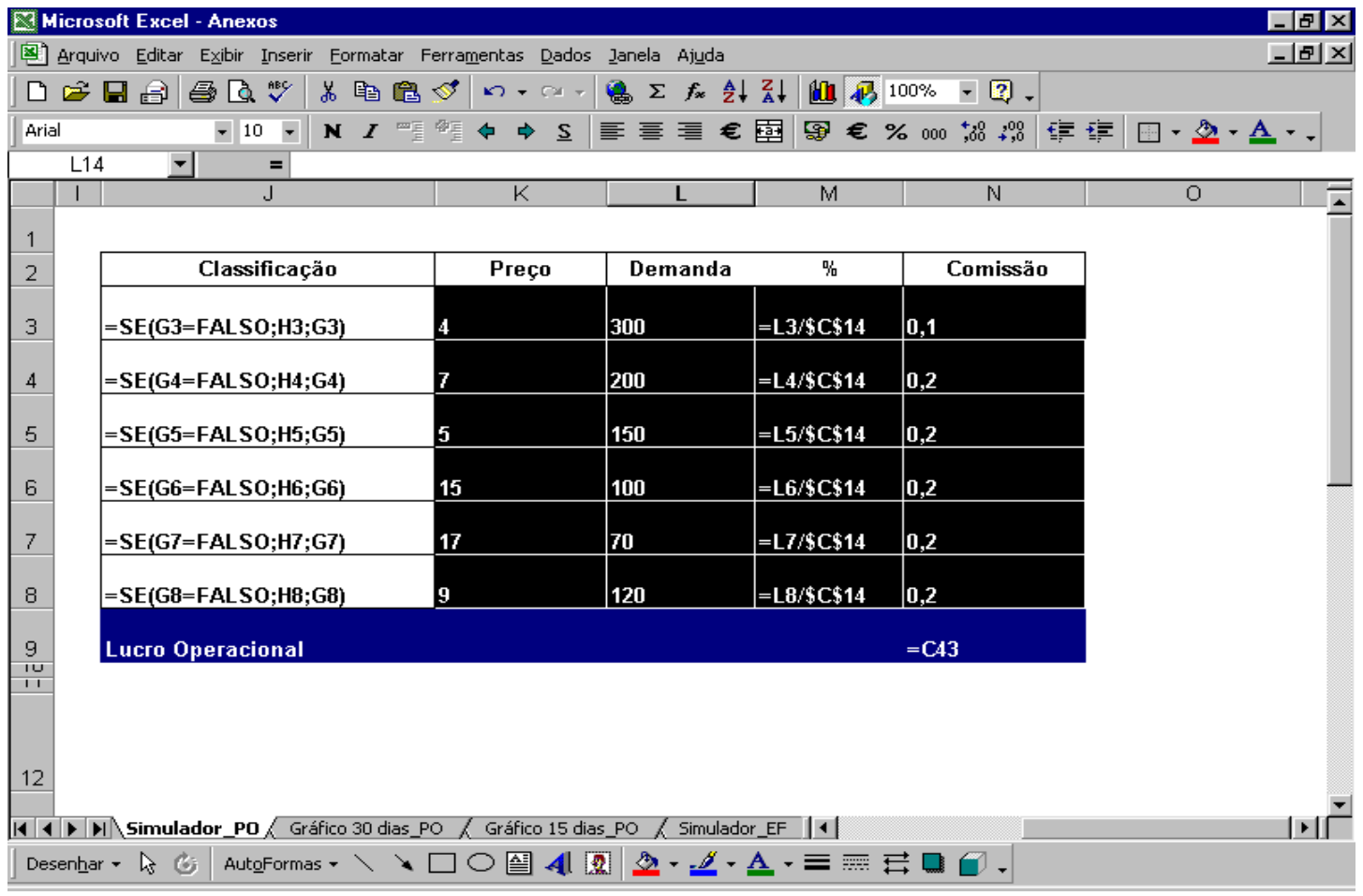


Custo-volume-lucro como ferramenta de gestão em uma empresa de serviços

Flávia Zoboli Dalmacio, Luciene Laurett Rangel, Venina de Almeida Lopes, Aridelmo José Campanharo

Teixeira.

Nota: Por limitação do excel, a fórmula lógica "se", da coluna "classificação", precisou ser desmembrada em três (coluna "J" do apêndice 2 e colunas "G" e "H" do apêndice 3).

Apêndice 3: Demonstração da Fórmula Lógica da Coluna "J" (Classificação).

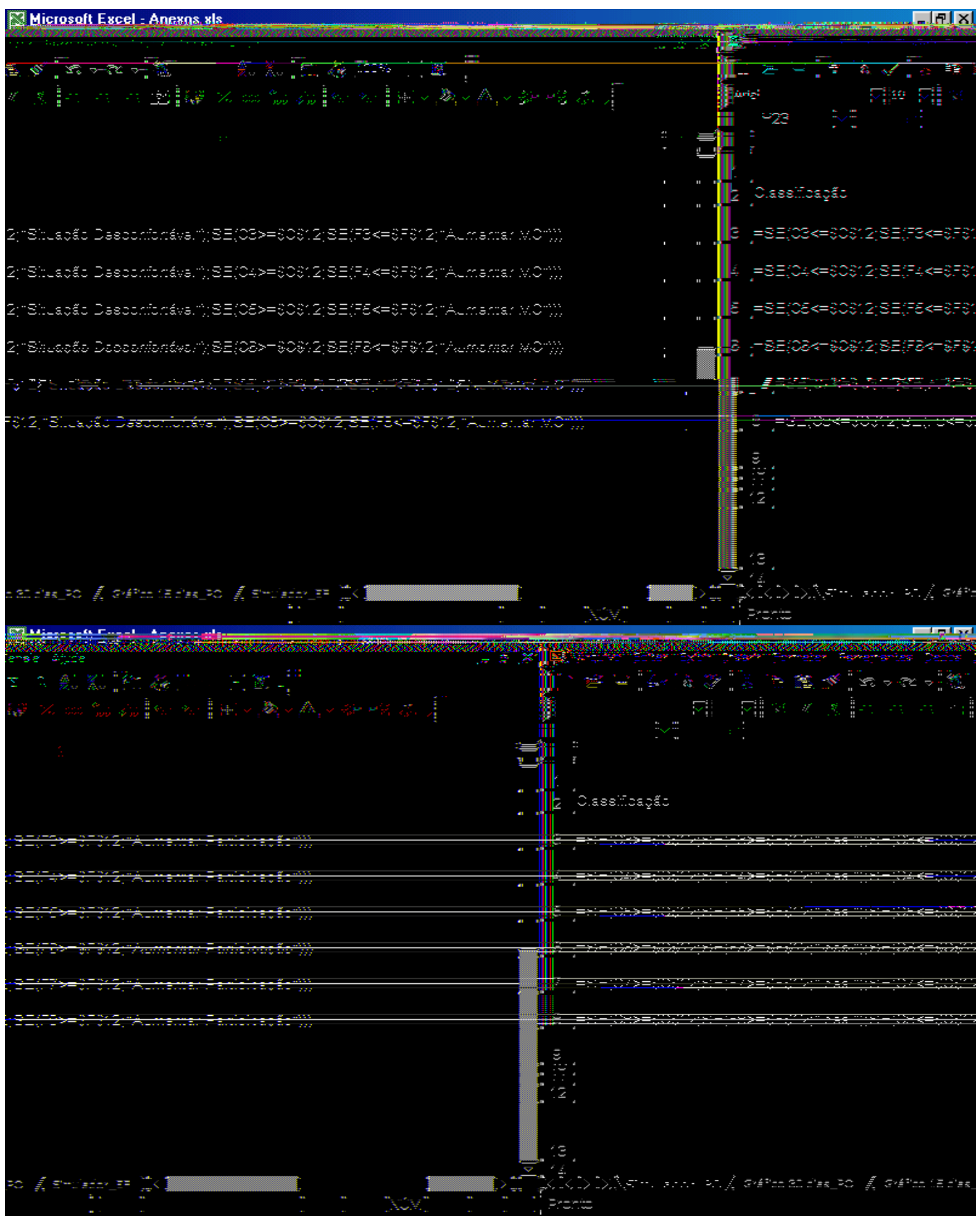


Custo-volume-lucro como ferramenta de gestão em uma empresa de serviços

Flávia Zoboli Dalmacio, Luciene Laurett Rangel, Venina de Almeida Lopes, Aridelmo José Campanharo

Teixeira.

Apêndice 4: Parte 3 - Demonstração do Resultado e Break-Even Point.

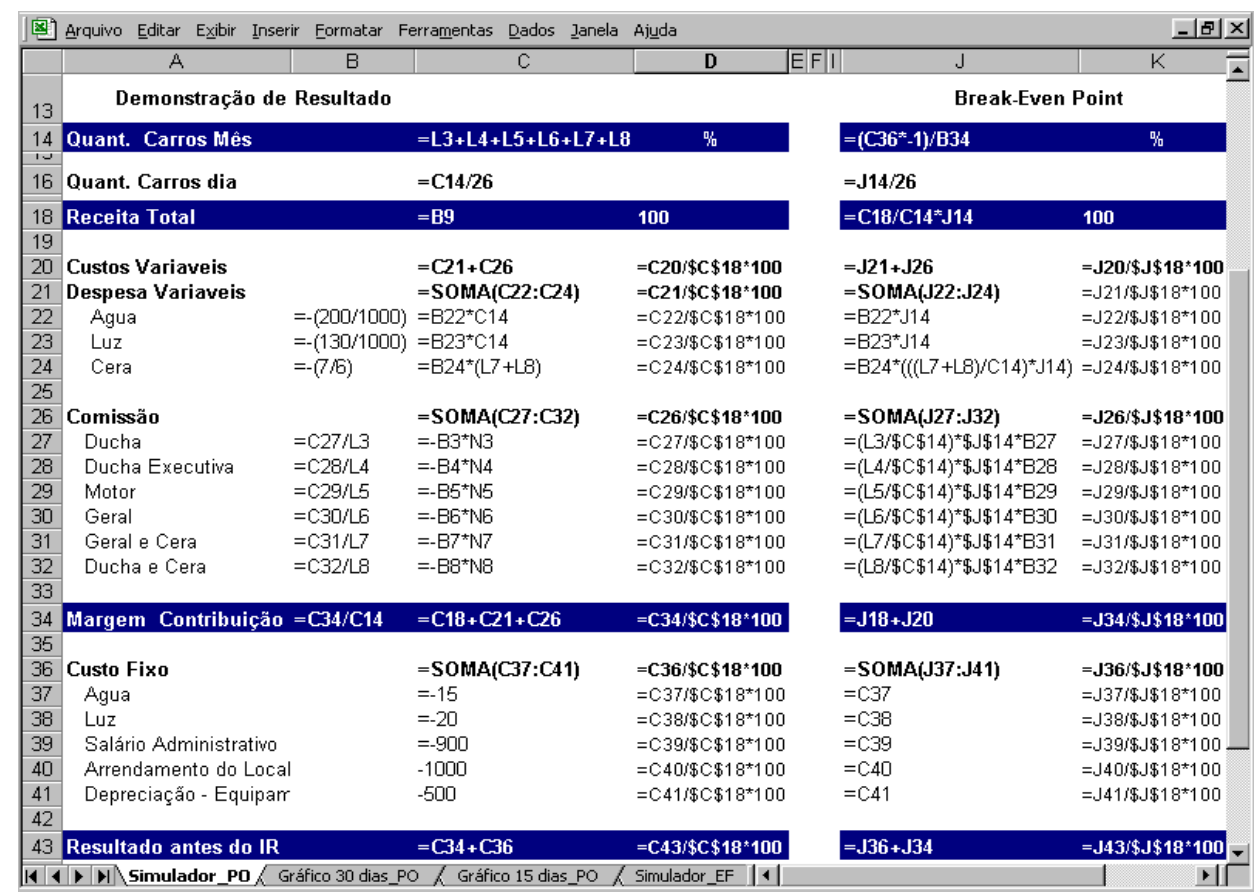

Apêndice 5: Análise da Performance Operacional de Vendas.

\begin{tabular}{|c|c|c|c|c|c|c|c|c|c|c|c|c|}
\hline \multirow[b]{3}{*}{ Serviços } & \multirow{2}{*}{\multicolumn{3}{|c|}{ Quantidade de Carros }} & \multirow{2}{*}{\multicolumn{6}{|c|}{$\begin{array}{l}\mathrm{D} / \mathrm{A} / \mathrm{E} / \mathrm{B} \\
\text { Preço unitário } \\
\end{array}$}} & \multirow{2}{*}{\multicolumn{3}{|c|}{$\frac{\mathrm{C}^{*} \mathrm{G}}{\mathrm{Aná}^{\mathrm{B}^{*} \mathrm{ise}}}$}} \\
\hline & & & & & & & & & & & & \\
\hline & $\begin{array}{l}\text { Real } \\
\text { (A) }\end{array}$ & $\begin{array}{c}\text { Plano } \\
\text { (B) }\end{array}$ & $\begin{array}{l}\text { Var. } \\
\text { (C) }\end{array}$ & $\begin{array}{l}\text { Real } \\
\text { (D) }\end{array}$ & $\begin{array}{l}\text { Plano } \\
\text { (E) }\end{array}$ & $\begin{array}{l}\text { Var. } \\
(\mathrm{F})\end{array}$ & $\begin{array}{l}\text { Real } \\
(G)\end{array}$ & $\begin{array}{c}\text { Plano } \\
(\mathrm{H})\end{array}$ & $\begin{array}{l}\text { Var. } \\
\text { (I) }\end{array}$ & \begin{tabular}{c|} 
Volume \\
Mix \\
(1)
\end{tabular} & $\begin{array}{c} \\
\text { Preço } \\
\text { (2) }\end{array}$ & Total \\
\hline Ducha & 263 & 300 & (37) & 921 & 1.200 & (280) & 3,50 & 4,00 & $(0,50)$ & (130) & (150) & (280) \\
\hline Ducha Executiva & 150 & 200 & (50) & 900 & 1.400 & $(500)$ & 6,00 & 7,00 & $(1,00)$ & (300) & (200) & (500) \\
\hline Motor & 100 & 150 & (50) & 500 & 750 & (250) & 5,00 & 5,00 & 0,00 & (250) & 0 & (250) \\
\hline Geral & 150 & 100 & 50 & 2100 & 1.500 & 600 & 14,00 & 15,00 & $(1,00)$ & 700 & (100) & 600 \\
\hline Geral e Cera & 122 & 70 & 52 & 1.952 & 1.190 & 762 & 16,00 & 17,00 & $(1,00)$ & 832 & (70) & 762 \\
\hline Ducha e Cera & 108 & 120 & (12) & 1.296 & 1.080 & 216 & 12,00 & 9,00 & 3,00 & (144) & 360 & 216 \\
\hline TOTAL & 893 & 940 & (47) & 7.669 & 7.120 & 549 & 8,59 & 7,57 & 1,01 & 709 & $(160)$ & 549 \\
\hline
\end{tabular}

Apêndice 6: Análise da Performance Operacional de Custos. 
Custo-volume-lucro como ferramenta de gestão em uma empresa de serviços Flávia Zoboli Dalmacio, Luciene Laurett Rangel, Venina de Almeida Lopes, Aridelmo José Campanharo Teixeira.

\begin{tabular}{|c|c|c|c|c|c|c|c|c|c|c|c|c|}
\hline \multirow[b]{3}{*}{ Serviços } & & & & & & & $7^{\sqrt{J / A}}$ & K/B & & $>-C^{*}$ & $7 \mathrm{~B}^{*} \mathrm{C}$ & \\
\hline & \multicolumn{3}{|c|}{ Quantidade de Carros } & \multicolumn{3}{|c|}{ Custo Serv. Prestados } & \multicolumn{3}{|c|}{ Preço de Custo unitário } & \multicolumn{3}{|c|}{ Análise dos Custos } \\
\hline & $\begin{array}{l}\text { Real } \\
\text { (A) }\end{array}$ & $\begin{array}{l}\text { Plano } \\
\text { (B) }\end{array}$ & $\begin{array}{l}\text { Var. } \\
\text { (C) }\end{array}$ & $\begin{array}{l}\text { Real } \\
\text { (J) }\end{array}$ & $\begin{array}{l}\text { Plano } \\
\text { (K) }\end{array}$ & $\begin{array}{l}\text { Var. } \\
\text { (L) }\end{array}$ & $\begin{array}{l}\text { Real } \\
\text { (M) }\end{array}$ & $\begin{array}{l}\text { Plano } \\
\text { (N) }\end{array}$ & $\begin{array}{l}\text { Var. } \\
\text { (O) }\end{array}$ & $\begin{array}{l}\text { Volume } \\
\text { Mix } \\
\text { (3) }\end{array}$ & $\begin{array}{l}\text { Preço } \\
\text { (4) }\end{array}$ & Total \\
\hline Ducha & 263 & 300 & (37) & 263 & 219 & (44) & 1,00 & 0,73 & $(0,27)$ & 37 & (81) & (44) \\
\hline Ducha Executiva & 150 & 200 & (50) & 210 & 346 & 136 & 1,40 & 1,73 & 0,33 & 70 & 66 & 136 \\
\hline Motor & 100 & 150 & (50) & 124 & 200 & 76 & 1,24 & 1,33 & 0,09 & 62 & 14 & 76 \\
\hline Geral & 150 & 100 & 50 & 402 & 333 & (69) & 2,68 & $3, \mathbf{3 3}$ & 0,65 & (134) & 65 & (69) \\
\hline Geral e Cera & 122 & 70 & 52 & 539 & 343 & (196) & 4,42 & 4,90 & 0,48 & (230) & 34 & (196) \\
\hline Ducha e Cera & 108 & 120 & (12) & 408 & 396 & (12) & 3,78 & 3,30 & $(0,48)$ & 45 & (58) & (12) \\
\hline TOTAL & 893 & 940 & (47) & 1.946 & 1.836 & (110) & 2,18 & 1,95 & $(0,23)$ & (149) & 40 & (110) \\
\hline
\end{tabular}

i Os procedimentos de como se obter os valores para as análises de vendas e custos, encontram-se nos apêndices 5 e 6 , possibilitando um maior detalhamento dos dados. 